\title{
Giant Enhancement of the Thermo-Particle Transport Figure of Merit and Breakdown of the Wiedemann-Franz Law in Unitary Fermi Gases
}

\author{
Xinloong Han, ${ }^{1,2}$ Boyang Liu,, , and Jiangping $\mathrm{Hu}^{1,2,4, \text { 丹 }}$ \\ ${ }^{1}$ Beijing National Laboratory for Condensed Matter Physics, \\ and Institute of Physics, Chinese Academy of Sciences, Beijing 100190,China \\ ${ }^{2}$ University of Chinese Academy of Sciences, Beijing 100049, China \\ ${ }^{3}$ Institute of Theoretical Physics, Beijing University of Technology Beijing 100124, China \\ ${ }^{4}$ Collaborative Innovation Center of Quantum Matter, Beijing, China
}

(Dated: May 31, 2021)

\begin{abstract}
We theoretically investigate the thermo-particle transport properties of an unitary Fermi gas between two reservoirs connected by a quantum point contact. We find several distinguished properties that are qualitatively different from those of weak or non-interacting gas systems. The particle transport figure of merit is drastically enhanced in the unitary regime and it increases as the transmission coefficient increases, exactly opposite to the behavior in the weak or non-interacting gas systems. The Lorentz number violates the Wiedemann-Franz law, demonstrating the breakdown of Fermi liquid. These transport properties are the hallmarks of the unitary Fermi gas and are attributed to the existence of preformed Cooper pairs.
\end{abstract}

Introduction. Thermo-transport and thermoelectricity are critical physical properties of solid state systems [1, 2]. Recently, the study of the transport in cold atom systems has attracted tremendous research attentions 3 - 17]. Of particular interests is the realization of thermo-particle transport of cold atoms [4, 10]. Although atoms are charge-neutral, the conversion between the heat and particle current, as an analogy to thermoelectricity, can be a potential important application of the ultracold atom systems.

Particularly, in experiment, the transport with twoterminal setup realized by Esslinger's group at ETH is a milestone in quantum simulation [3 5, 7 9]. In 2013 Esslinger's group realized the conversion of heat and matter in a non-interacting fermionic cold atom gas and investigated the efficiency of energy conversion by controlling the geometry and disorder strength [4]. The experiment paved a road to study the thermo-particle transport effect in cold atom physics. Furthermore, most recently, Esslinger's group studied the thermo-particle transport effect in a unitary Fermi gas in the superfluid state and various transport coefficients were measured 10]. The experiment suggested that the Wiedemann-Franz law was not valid in the unitary case. In the unitary regime, the particle conductivity has been investigated both experimentally [3, 8] and theoretically [18 21]. However the thermo-particle transport has not been well studied theoretically.

In this Letter, we study the thermo-particle transport in the normal state of a Fermi gas with a tunable interaction. We calculate several thermo-transport quantities, including particle conductivity $G$, thermal conductivity $G_{T}$, Seeback coefficient $\alpha$ and the dimensionless figure of merit $Z T=\frac{G \alpha^{2} T}{G_{T}}$, which is known to be the most important quantity in determining the energy conversion efficiency between heat and particle currents. We implement the Nozières and Schmitt-Rink (NSR) scheme to consider the effect of preformed Cooper pairs 22, 23. and calculate thermal transport quantities by Keldysh formulism [24, 25]. We find that the thermo-transport in the unitary region is characterized by several distinguished features which are absent or behave completely opposite to those in the weak interacting regime. First, the figure of merit has a giant enhancement near the superfluid transition temperature in the unitary Fermi gas. Second, increasing the transmission coefficient of the point contact can increase the figure of merit. This behavior is exactly opposite to the one in the weak interaction Fermi gas, in which the figure of merit decreases as the transport ability of QPC increases [4]. Finally, the Lorentz number $L=\frac{G_{T}}{T G}$ of unitary Fermi gas violates the Wiedemann-Franz law [26, 27], which signals the breakdown of the Fermi liquid. The deviation from the Wiedemann-Franz law also increases as the transmission coefficient increases. All these thermo-transport properties of the unitary Fermi gas can be attributed to the existence of preformed Cooper pairs above the superfluid transition temperature. Our calculation not only explains the observed experimental results, but also can be extended to determine the possible existence of preformed Cooper pairs in superconducting materials.

Model and method.- - We study the system with a twoterminal setup. Two reservoirs are connected by a quantum point contact, which is formed using high-resolution lithography [5, 7, 8]. A laser beam working as an attractive gate potential is shone on the QPC region. The number of the open channels can be changed by tuning the gate potential 28. In this work, we limit our calculation for the single open channel case. Particle and heat currents are generated by imposing temperature and chemical potential differences between the two reservoirs. The Hamiltonian of the system can be cast in three parts as the following(setting $\hbar=1$ and $k_{B}=1$ ) 29 31],

$$
\hat{H}=\hat{H}_{L}+\hat{H}_{R}+H_{T},
$$


where $\hat{H}_{L}\left(\hat{H}_{R}\right)$ is the Hamiltonian of the left(right) reservoir and is written as

$$
\begin{aligned}
\hat{H}_{j}= & \int d^{3} \mathbf{r}\left\{\sum_{\sigma} \hat{\psi}_{j \sigma}^{\dagger}(\mathbf{r})\left(-\frac{\nabla^{2}}{2 m}-\mu_{j}\right) \hat{\psi}_{j \sigma}(\mathbf{r})\right. \\
& \left.-g \hat{\psi}_{j \uparrow}^{\dagger}(\mathbf{r}) \hat{\psi}_{j \downarrow}^{\dagger}(\mathbf{r}) \hat{\psi}_{j \downarrow}(\mathbf{r}) \hat{\psi}_{j \uparrow}(\mathbf{r})\right\} .
\end{aligned}
$$

The operator $\hat{\psi}_{j \sigma}(\mathbf{r})$ and $\hat{\psi}_{j \sigma}^{\dagger}(\mathbf{r})$ describe the annihilation and creation of a fermion atom with spin $\sigma$ in the $j$-th reservoir with $j=L, R . m$ is the mass of fermions and $\mu_{j}$ is the chemical potential of the $j$-th reservoir. The parameter $g$ is the bare interaction strength between the spin up and spin down atoms. It's related to the $s$ wave scattering length $a_{s}$ by the renormalization relation $\frac{1}{g}=-\frac{m}{4 \pi a_{s}}+\int \frac{d^{3} \mathbf{k}}{(2 \pi)^{3}} \frac{1}{2 \epsilon_{\mathbf{k}}}$, where $\epsilon_{\mathbf{k}}=\mathbf{k}^{2} / 2 m$. We study this model above the superfluid transition temperature under the NSR scheme, where the effect of preformed Cooper pairs is taken into account by calculating the ladder diagram as shown in Fig. 10 (b).

The Hamiltonian $\hat{H}_{T}$ describes the tunneling of fermions between the two reservoirs and is written as

$$
\hat{H}_{T}=\sum_{\sigma} t \hat{\psi}_{L \sigma}^{\dagger}(0) \hat{\psi}_{R \sigma}(0)+h . c . .
$$

Here we assume this process only occurs at a single point $\mathbf{x}=0$. In the single open channel case, the transport properties through the QPC is assumed to be controlled by the parameter $t$, the tunneling amplitude.

In the realistic cold atom experiment one reservoir may be heated up by a laser beam. The heat and particle currents are generated between two reservoirs by temperature bias $\Delta T=T_{L}-T_{R}$ or chemical potential bias $\Delta \mu=\mu_{L}-\mu_{R}$. They are defined as $I_{Q} \equiv \frac{1}{2}\left\langle\frac{\partial\left(\hat{E}_{R}-\hat{E}_{L}\right)}{\partial t}\right\rangle$ and $I_{N} \equiv \frac{1}{2}\left\langle\frac{\partial\left(\hat{N}_{R}-\hat{N}_{L}\right)}{\partial t}\right\rangle$, with $\hat{E}_{j} \equiv \sum_{\sigma} \int d^{3} \mathbf{r} \hat{\psi}_{j \sigma}^{\dagger}(t, \mathbf{r}) i \partial_{t} \hat{\psi}_{j \sigma}(t, \mathbf{r})$ and $\hat{N}_{j} \equiv$ $\sum_{\sigma} \int d^{3} \mathbf{r} \hat{\psi}_{j \sigma}^{\dagger}(t, \mathbf{r}) \hat{\psi}_{j \sigma}(t, \mathbf{r})$ being the energy operator and number operator of the $j$-th reservoir respectively. Using the Keldysh formulaism [24, 25], the average $\langle\ldots\rangle$ in above expressions is calculated over the time-evolving manybody state on a closed time contour. In the linear response regime, we consider the small temperature and chemical potential bias, $\Delta T$ and $\Delta \mu$. The currents can be calculated up to the first order of $\Delta T$ and $\Delta \mu$ as the following,

$$
\left(\begin{array}{l}
I_{N} \\
I_{Q}
\end{array}\right)=\left(\begin{array}{ll}
L_{11} & L_{12} \\
L_{21} & L_{22}
\end{array}\right)\left(\begin{array}{c}
\Delta \mu \\
\Delta T
\end{array}\right)
$$

where $L_{i j}$ is known as the Lorentz matrix, the elements are given by

$$
\begin{aligned}
& L_{11}=-\int \frac{d \omega}{2 \pi}\left\{2 \mathcal{F}^{2}(\omega) \frac{\partial n_{F}}{\partial \omega}+4 \mathcal{B}^{2}(\omega) \frac{\partial n_{B}}{\partial \omega}\right\}, \\
& L_{12}=-\int \frac{d \omega}{2 \pi} \frac{\omega}{\bar{T}}\left\{2 \mathcal{F}^{2}(\omega) \frac{\partial n_{F}}{\partial \omega}+2 \mathcal{B}^{2}(\omega) \frac{\partial n_{B}}{\partial \omega}\right\}, \\
& L_{21}=-\int \frac{d \omega}{2 \pi} \omega\left\{2 \mathcal{F}^{2}(\omega) \frac{\partial n_{F}}{\partial \omega}+2 \mathcal{B}^{2}(\omega) \frac{\partial n_{B}}{\partial \omega}\right\},
\end{aligned}
$$

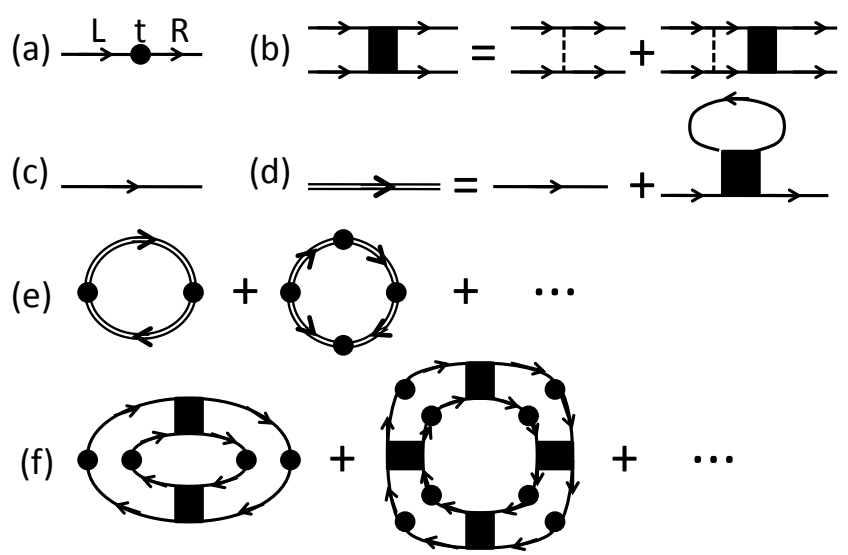

FIG. 1. The Feynman diagrams of (a), the tunneling term; (b), renormalized interaction under the NSR scheme; (c), free propagator of fermion; (d), full propagator of fermion under the NSR scheme; (e), current contributed by fermions; (f), current contributed by preformed Cooper pairs.

$$
L_{22}=-\int \frac{d \omega}{2 \pi} \frac{\omega^{2}}{\bar{T}}\left\{2 \mathcal{F}^{2}(\omega) \frac{\partial n_{F}}{\partial \omega}+\mathcal{B}^{2}(\omega) \frac{\partial n_{B}}{\partial \omega}\right\} .
$$

The elements $L_{12}$ and $L_{21}$ are related by the Onsager relation as $L_{21}=\bar{T} L_{12}\left[33\right.$, 34], where $\bar{T}=\left(T_{L}+T_{R}\right) / 2$. $n_{F}(\omega)=\frac{1}{\exp (\beta \omega)+1}$ and $n_{B}(\omega)=\frac{1}{\exp (\beta \omega)-1}$ are the Fermi and Bose distribution functions, where $\beta=1 / T$. In above expression one observes that both particle and heat currents include two parts. One is from fermions and the other one is from bosons, which is the contribution of the preformed Cooper pairs. These two kinds of contributions can be summarized by the Feynman diagrams in Fig. 1 (e) and (f). Both of them are infinite summation to all the order of $t$. The function $\mathcal{F}(\omega)$ and $\mathcal{B}(\omega)$ are expressed as $\mathcal{F}(\omega)=\frac{2 \mathcal{T} A_{F}(\omega) / A_{F}(0)}{\mid 1+\mathcal{T}^{2}\left(A_{F}(\omega)\right)^{2} /\left(A_{F}(0)\right)^{2}}$ and $\mathcal{B}(\omega)=\frac{\pi \mathcal{T}^{2} \sqrt{\beta} A_{B}(\omega)}{\mid 1-\mathcal{T}^{4} \beta\left(\operatorname{Re} G_{B}(\omega)\right)^{2} / 4}$, the Taylor expansion of which with respect to $\mathcal{T}$ manifestly demonstrates the infinite summation of graphs in Fig. 1 (e) and (f). Here we define a dimensionless transmission amplitude $\mathcal{T}=\pi t A_{F}(0)$. With the recently developed technique of scanning gate microscope in cold atom experiments, the effective tunneling amplitude $\mathcal{T}$ can be tuned continuously from 0 to 1 [9, 11, 32]. The spectral functions of fermions and Cooper pairs are given by

$$
\begin{aligned}
& A_{F}(\omega)=-\frac{1}{\pi} \int \frac{d^{2} \mathbf{k}}{(2 \pi)^{2}} \operatorname{Im}\left(\frac{1}{\omega+i 0^{+}-\epsilon_{\mathbf{k}}+\mu-\Sigma_{F}(\omega, \mathbf{k})}\right), \\
& A_{B}(\omega)=-\frac{1}{\pi} \int \frac{d^{2} \mathbf{q}}{(2 \pi)^{2}} \operatorname{Im}\left(G_{B}\left(\omega+i 0^{+}, \mathbf{q}\right)\right),
\end{aligned}
$$

where $\Sigma_{F}(\omega, \mathbf{k})$ is the self energy of the Fermions $\Sigma_{F}(\omega, \mathbf{k})=-\frac{1}{\beta} \sum_{\omega_{m}} \int \frac{d^{3} \mathbf{p}}{(2 \pi)^{3}} \frac{G_{B}\left(i \omega_{m}, \mathbf{p}\right)}{\left[\omega+i 0^{+}-i \omega_{m}+(\mathbf{p}-\mathbf{k})^{2} / 2 m-\mu\right]}$, and the propagator of Cooper pair is $G_{B}\left(i \omega_{m}, \mathbf{k}\right)=$ $\left(-\frac{m}{4 \pi a_{s}}-\int \frac{d^{3} \mathbf{p}}{(2 \pi)^{3}}\left[\frac{1-n_{F}\left(\epsilon_{\mathbf{p}}-\mu_{j}\right)-n_{F}\left(\epsilon_{\mathbf{p}-\mathbf{k}}-\mu\right)}{-i \omega_{m}+\epsilon_{\mathbf{p}}+\epsilon_{\mathbf{p}}-\mathbf{k}-2 \mu}-\frac{1}{2 \epsilon_{\mathbf{p}}}\right]\right)^{-1}$. 

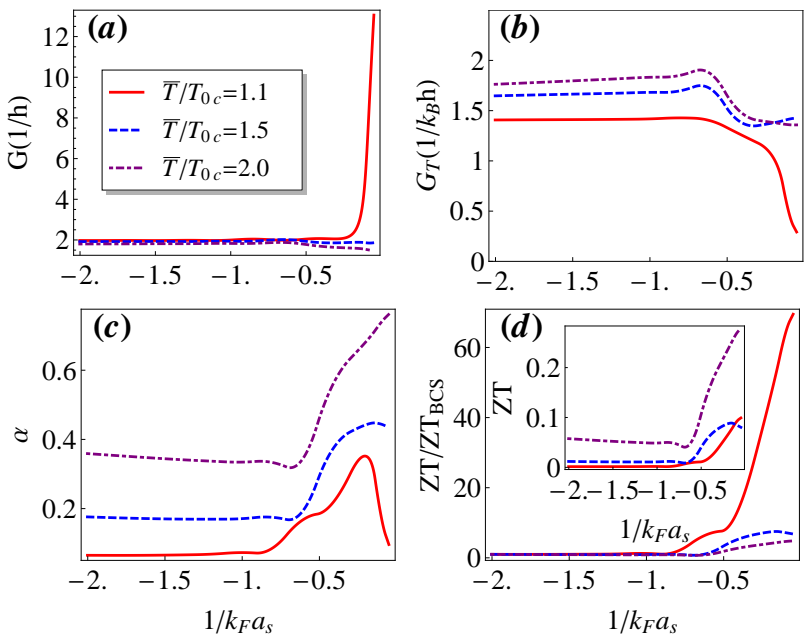

FIG. 2. (Color online) The thermo-particle coefficients (a), the conductance $G$; (b), the thermal conductance $G_{T}$; (c), the Seebeck coefficient $\alpha$, and (d), the ratio of figure of merit $Z T / Z T_{B C S}$ as functions of $1 / a_{s} k_{F}$. The red, blue dashed and purple dashed dotted lines are for temperature $T / T_{0 c}=$ $1.1,1.5,2$, respectively, where $T_{0 c}$ is the critical temperature at the unitarity. The transmission coefficient is set as $\mathcal{T}=1$.

In the realistic cold atom experiments, the QPC structure is not directly connected to the three-dimensional reservoirs. Instead it's connected to a two-dimensional region first, which is formed by the repulsive potential of a TEM01-like mode of a laser [5, 7, 8]. Hence, the integrations of momenta in the spectral function of Eq.(6) is two-dimensional. As illustrated in Ref. [20] the low dimensional structure is very important for the enhancement of the conductance.

Usually, the currents in Eq. (44) are expressed in terms of the thermo-particle coefficients as the following,

$$
\left(\begin{array}{c}
I_{N} \\
I_{Q}
\end{array}\right)=G\left(\begin{array}{cc}
1 & \alpha \\
\bar{T} \alpha & \bar{T}\left(L+\alpha^{2}\right)
\end{array}\right)\left(\begin{array}{c}
\Delta \mu \\
\Delta T
\end{array}\right)
$$

where the conductance $G$, Seebeck coefficient $\alpha$ and the Lorentz number $L$ are defined as $G=L_{11}, \alpha=L_{12} / L_{11}$, $L=L_{22} / L_{11}-\left(L_{12} / L_{11}\right)^{2}$ and $\bar{T}=\left(T_{L}+T_{R}\right) / 2$. Furthermore the thermal conductivity is defined as $G_{T}=\bar{T} G L$. Then the figure of merit can be calculated as

$$
Z T=\frac{G \alpha^{2} \bar{T}}{G_{T}}=\frac{L_{12} L_{21}}{L_{22} L_{11}-L_{12} L_{21}} .
$$

Enhanced figure of merit.-To investigate how the preformed Cooper pairs affect the figure of merit, we fix the temperature $\bar{T}$ slightly above the critical temperature $T_{0 c}$ at $1 / a_{s} k_{F}=0$, which is $T_{0 c}=0.218 T_{F}$ in the calculation of NSR, and study the variation of thermal coefficients with respect to the interaction strength. The system becomes closer to its critical temperature when $1 / a_{s} k_{F}$ increases from the BCS limit to the unitarity. Consequently, the effect of preformed Cooper pairs is much stronger at the unitarity than at the BCS limit.
In Fig 2(a), the conductance is shown to be significantly enhanced when the unitarity is approached at a temperature very close to $T_{0 c}$. Due to the divergence of the Bose distribution function $1 /(\exp (\beta \omega)-1)$ when the energy $\omega$ approaches 0 , the preformed Cooper pairs can generate a large contribution to the conductance between the two reservoirs [20]. At the BCS limit, the conductance obeys the calculation of Landauer-Büttiker formula [35, 36], $G=2 / h$ for one open channel case.

In contrast to the enhancement of particle conductance, the thermal conductance drops rapidly as one approaches the unitary regime as illustrated in Fig 2 (b). The strong separation of particle and thermal transport timescales has been observed in the experiment of Ref. [10], where they found the thermal conductance $G_{T}$ is roughly one order of magnitude smaller than the noninteracting case. Our calculation is consistent with their results.

The Seebeck coefficient $\alpha$ demonstrates a nonmonotonic behavior as a function of $1 / a_{s} k_{F}$ as shown in Fig. 22 (c). The drop of $\alpha$ around the unitarity is due to the large enhancement of $G$ since $\alpha=L_{12} / G$. Although the Seebeck coefficient drops in the unitary regime, it can not overcome the combined effect of significantly increased conductance and decreased thermal conductance. The combined effect results in an enhanced figure of merit in the unitary Fermi gas. In Fig. 2 (d) we compare ZT for different $1 / a_{s} k_{F}$ with $\mathrm{ZT}_{B C S}$, which is the figure of merit at $1 / a_{s} k=-2$. At the temperature $T / T_{0 c}=1.1$, $Z T / Z T_{B C S}$ increases by about two order of magnitude at the unitarity case. As the temperature increases, the enhancement becomes weaker and weaker due to the decrease of the preformed Cooper pair fraction.

The variation of the figure of merit by tuning the transmission amplitude $\mathcal{T}$ - Recently a new technique in cold atom experiment has been developed, with which the transmission coefficient $\mathcal{T}$ can be continuously tuned from 0 to 1 by imprinting a mesoscopic potential or a lattice into the QPC tunneling channel [9, 11, 32]. Here we investigate the variation of the transport coefficients and the figure of merit by tuning $\mathcal{T}$. The Fig $3(\mathrm{~d})$ shows that $Z T$ increases as the $\mathcal{T}$ decreases for temperature $T / T_{0 c}=1.5$ and 2 . In the experiment to study the thermo-particle effect of non-interacting Fermi gas [4], it has been shown that the figure of merit increases as the disorder strength becomes stronger. Large disorder strength suppresses the transport ability. Hence, the two results are consistent with each other. However, as the temperature decreases to $T / T_{0 c}=1.1$ and the effect of preformed Cooper pairs becomes profound, the figure of merit $Z T$ presents a different behavior from the cases of $T / T_{0 c}=1.5$ and 2. Namely, $Z T$ increases as $\mathcal{T}$ approaches 1. In Fig. 3 (b) and (c) one observes that $G_{T}$ increases and $\alpha$ decreases as $\mathcal{T}$ increases, which could result in the decrease of $Z T$. However, as shown in Fig. 3 (a) $G$ is significantly enhanced as $\mathcal{T}$ increases for $T / T_{0 c}=1.1$. 

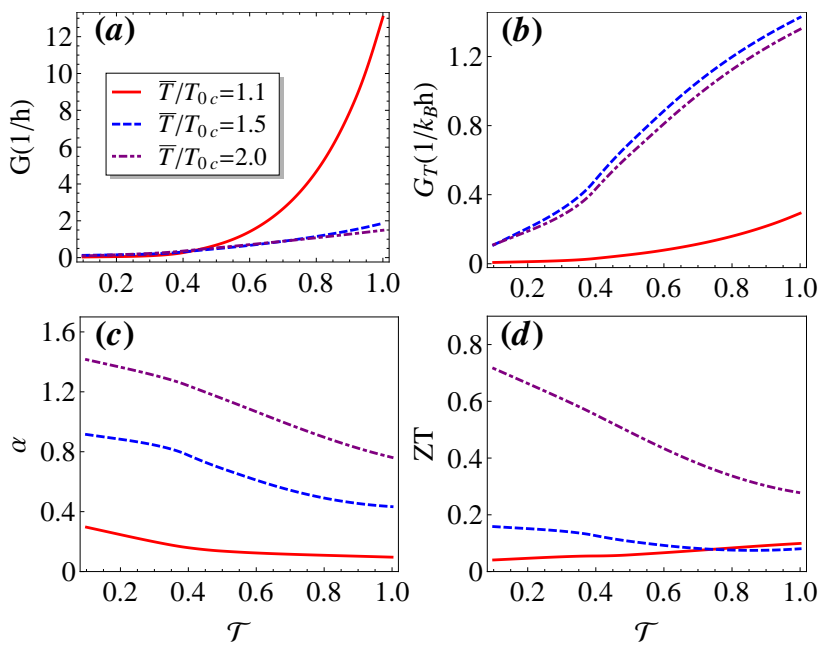

FIG. 3. (Color online) The thermo-particle coefficients (a), the conductance $G$; (b), the thermal conductance $G_{T}$; (c), the Seebeck coefficient $\alpha$, and (d), the figure of merit $Z T$ at $1 / a_{s} k_{F}=0$ as functions of the transmission coefficient $\mathcal{T}$. The red, blue dashed and purple dashed dotted lines are for temperature $T / T_{0 c}=1.1,1.5,2$, respectively.

Eventually the behavior of $G$ becomes the dominant factor and results in the increase of $Z T$.

Violation to the Wiedemann-Franz law.-In Fermi liquid, the Lorentz number $L$ takes a universal value $L_{0}=$ $\pi^{2} / 3$ at low temperature. This is known as WiedemannFranz law 26, 27]. The violation to the Wiedemann-Franz law indicates a non-Fermi liquid behavior. In Fig 4 (a), the Lorentz number is shown to be close to the universal value $L_{0}$ at the BCS limit at low temperature $T / T_{0 c}=1.1$. However, it drops rapidly in the unitary regime, which is a clear signal of the breakdown of Fermi liquid at the unitary regime.

Next, we investigate the variation of the Lorentz number at $1 / a_{s} k_{F}=0$ with respect to the transmission coefficient $\mathcal{T}$. In Fig 4 (b) it is demonstrated that $L$ increases and approaches the universal value $L_{0}$ as $\mathcal{T}$ decreases. We can conclude that the effect of preformed Cooper pairs becomes weaker as $\mathcal{T}$ decreases. Namely, for a small transmission coefficient, the transport properties of unitary Fermi gases are closer to the Fermi liquid compared with the case of a large transmission coefficient.

Concluding Remark.-We theoretically investigate the thermo-particle transport properties of an unitary Fermi gas. We find that the particle transport figure of merit can be enhanced dramatically in the unitary regime, comparing to the weak interaction regime. The WiedemannFranz law is strongly violated as well. The underlying physics can be attributed to the existence of preformed Cooper pairs. These results not only explain recent experimental results but also suggest a new way to determine possible preformed Cooper pairs in other systems.

Acknowledgements We thank Hui Zhai and Shizhong
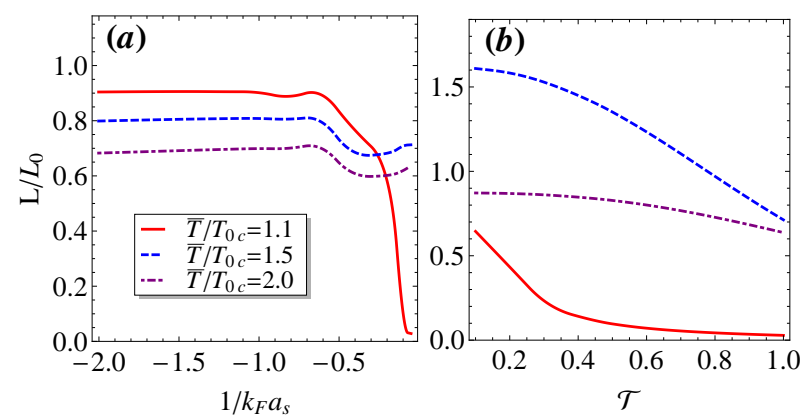

FIG. 4. (Color online) (a), the Lorentz number as functions of $1 / a_{s} k_{F}$ for $\mathcal{T}=1$; (b), the Lorentz number as functions of $\mathcal{T}$ for $1 / a_{s} k_{F}=0$. The red, blue dashed and purple dashed dotted lines are for temperature $T / T_{0 c}=1.1,1.5,2$, respectively.

Zhang for very helpful discussions. The work is supported by the Ministry of Science and Technology of China 973 program (No. 2015CB921300), National Science Foundation of China (Grant No. NSFC-1190020, 11534014, 11334012), and the Strategic Priority Research Program of CAS (Grant No.XDB07000000).

* boyangleo@gmail.com

† jphu@iphy.ac.cn

[1] H. J. Goldsmid, Introduction to Thermoelectricity, Springer Series in Materials Science (Springer, Dordrecht,2009).

[2] D. MacDonald, Thermoelectricity: An Introduction to the Principles, Dover books on physics (Dover Publications, 2006).

[3] D. Stadler, S. Krinner, J. Meineke, J.-P. Brantut, and T, Esslinger, Nature 491, 736 (2012).

[4] J.-P. Brantut, C. Grenier, J. Meineke, D. Stadler, S. Krinner, C. Kollath, T. Esslinger, A. Georges, Science 342, 713 (2013).

[5] S. Krinner, D. Stadler, D Husmann, J. -P. Brantut, and T. Esslinger, Nature 517, 64 (2015).

[6] G. Valtolina, A. Burchianti, A. Amico, E. Neri, K. Xhani, J. A. Seman, A. Trombettoni, A. Smerzi, M. Zaccanti, M. Inguscio, and G. Roati, Science 350, 1505 (2015).

[7] D. Husmann, S. Uchino, S. Krinner, M. Lebrat, T. Giamarchi, T. Esslinger, and J.-P. Brantut, Science 350, 1498 (2015).

[8] S. Krinner, M. Lebrat, D. Husmann, C. Grenier, J.-P. Brantut, and T. Esslinger, Proc. Natl. Acad. Sci. 113, 8144 (2016).

[9] S. Häusler, S. Nakajima, M. Lebrat, D. Husmann, S. Krinner, T. Esslinger, and J.-P. Brantut, Phys. Rev. Lett. 119, 030403 (2017).

[10] D. Husmann, M. Lebrat, S. Häusler, arXiv:1803.00935 (2018).

[11] M. Lebrat, P. Grišins, D. Husmann, S. Häusler, L. Corman, T. Giamarchi, J.-P. Brantut, T. Esslinger, Phys. Rev. X 8, 011053 (2018). 
[12] A. Burchianti, F. Scazza, A. Amico, G. Valtolina, J. A. Seman, C. Fort, M. Zaccanti, M. Inguscio, and G. Roati, Phys. Rev. Lett. 120025302 (2018).

[13] A. Sommer, M. Ku, G. Roati, and M.W. Zwierlein, Nature 472, 201 (2011).

[14] A. B. Bardon, S. Beattie, C. Luciuk, W. Cairncross, D. Fine, N. S. Cheng, G. J. A. Edge, E. Taylor, S. Zhang, S. Trotzky, and J. H. Thywissen, Science 344, 722 (2014).

[15] M. Koschorreck, D. Pertot, E. Vogt, M. Kohl, Nat. Phys. 9, 405 (2013).

[16] C. Luciuk, S. Smale, F. Bottcher, H. Sharum, B. A. Olsen, S. Trotzky, T. Enss, and J. H. Thywissen, Phys. Rev. Lett. 118, 130405 (2017).

[17] G. Valtolina, F. Scazza, A. Amico, A. Burchianti, A. Recati, T. Enss, M. Inguscio, M. Zaccanti, and G. Roati, Nat. Phys. 13, 704 (2017).

[18] B. Liu, H. Zhai, and S. Zhang, Phys. Rev. A 90, 051602(R) (2014).

[19] M. Kanász-Nagy, L. Glazman, T. Esslinger, E. A. Demler, Phys. Rev. Lett. 117, 255302 (2016).

[20] B. Liu, H. Zhai, S. Zhang, Phys. Rev. A 95, 013623 (2017).

[21] S. Uchino, and M. Ueda, Phys. Rev. Lett. 118, 105303 (2017).
[22] P. Nozières and S. Schmitt-Rink, J. Low Temp. Phys. 59, 195 (1985).

[23] Y. Ohashi, and A. Griffin, Phys. Rev. Lett. 89, 130402 (2002).

[24] L. V. Keldysh, Sov. Phys. JETP 20, 1018 (1965).

[25] A. Kamenev, Field theory of non-equilibrium systems, Cambridge University Press, 2011.

[26] G. Wiedemann, and R. Franz, Ann. Phys. 89, 497 (1853).

[27] C. Kittel, Introduction to Solid State Physics, John Wiley and Sons, 2005.

[28] Y. V. Nazarov, and Y. M. Blanter, Quantum Transport: Introduction to Nanoscience, Cambridge University Press, 2009.

[29] J. C. Cuevas, A. Martín-Rodero, A. L. Yeyati, Phys. Rev. B 54, 7366 (1996).

[30] C. J. Bolech, T. Giamarchi, Phys. Rev. Lett. 92, 127001 (2004).

[31] C. J. Bolech, T. Giamarchi, Phys. Rev. B 71, 024517 (2005).

[32] J. Yao, B. Liu, M. Sun, H. Zhai, arXiv:1712.07125(2017).

[33] L. Onsager, Phys. Rev. 37, 405 (1931).

[34] L. Onsager, Phys. Rev. 38, 2265 (1931).

[35] R. Landauer, IBM J. Res. Develop. 1, 223 (1957).

[36] M. Büttiker, Y. Imry, R. Landauer, and S. Pinhas, Phys. Rev. B 31, 6207 (1985). 